\title{
3D GEOLOGICAL OUTCROP CHARACTERIZATION: AUTOMATIC DETECTION OF 3D PLANES (AZIMUTH AND DIP) USING LiDAR POINT CLOUDS
}

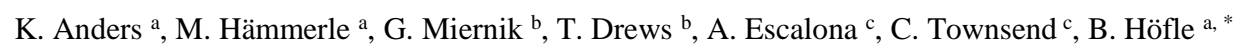 \\ a Institute of Geography, GIScience, LiDAR Research Group (LRG), Heidelberg University, Heidelberg, Germany - k.an- \\ ders@stud.uni-heidelberg.de, (haemmerle, hoefle)@uni-heidelberg.de \\ b Institute of Earth Sciences, Heidelberg University, Heidelberg, Germany - (georg.miernik, till.drews)@ geow.uni-heidelberg.de \\ ${ }^{c}$ Department of Petroleum Engineering, University of Stavanger, Stavanger, Norway - (alejandro.escalona, chris.townsend)@ uis.no
}

\section{Commission V, WG V/5}

KEY WORDS: LiDAR, Terrestrial laser scanning, 3D segmentation, Plane detection, Geological outcrop, Orientation, Strike

\begin{abstract}
:
Terrestrial laser scanning constitutes a powerful method in spatial information data acquisition and allows for geological outcrops to be captured with high resolution and accuracy. A crucial aspect for numerous geologic applications is the extraction of rock surface orientations from the data. This paper focuses on the detection of planes in rock surface data by applying a segmentation algorithm directly to a 3D point cloud. Its performance is assessed considering (1) reduced spatial resolution of data and (2) smoothing in the course of data pre-processing. The methodology is tested on simulations of progressively reduced spatial resolution defined by varying point cloud density. Smoothing of the point cloud data is implemented by modifying the neighborhood criteria during normals estimation. The considerable alteration of resulting planes emphasizes the influence of smoothing on the plane detection prior to the actual segmentation. Therefore, the parameter needs to be set in accordance with individual purposes and respective scales of studies. Furthermore, it is concluded that the quality of segmentation results does not decline even when the data volume is significantly reduced down to $10 \%$. The azimuth and dip values of individual segments are determined for planes fit to the points belonging to one segment. Based on these results, azimuth and dip as well as strike character of the surface planes in the outcrop are assessed. Thereby, this paper contributes to a fully automatic and straightforward workflow for a comprehensive geometric description of outcrops in 3D.
\end{abstract}

\section{INTRODUCTION}

Light detection and ranging (LiDAR), also known as laser scanning, is a method of rapid acquisition of high precision three-dimensional data. Its potential has been recognized in numerous fields of the Earth sciences and the use of this method has been growing rapidly over recent years (Höfle and Rutzinger, 2011; Buckley et al., 2010). Laser scanning technology can be conducted from different platforms, both airborne and terrestrial. When examining outcrops with generally vertical character, topographic information acquired by terrestrial laser scanning (TLS) platforms is preferred due to the advantageous perspective when compared to the rather plan view of terrain surfaces obtained by airborne survey (Sturzenegger and Stead, 2009). Furthermore, TLS enables higher levels of accuracy and point density (Beraldin et al., 2010).

The background of our research is the application of TLS in the study of geological outcrops, where it allows for a fast and consistent measurement of surfaces. Moreover, TLS enables mapping of otherwise difficult or dangerous to reach and inaccessible areas (Fisher et al., 2014). It is therefore attractive not only for research in the field of geology, but also for practical use in engineering and related fields, for example for geomechanical evaluation or determination of slope instabilities (Abellán et al., 2014; Fekete and Diederichs, 2013; Lato and Vöge, 2012). An essential component of outcrop characterization and important step for further analyses is the geometric description of the outcrop surface via azimuth and dip. The crucial part therein is the identification of planar surfaces in the unorganized raw point cloud data. Numerous established algorithms exist for point cloud segmentation, i.e. grouping of points based on specific criteria (Pfeifer et al., 2014; Barnea and Filin, 2013; Vosselmann and Klein, 2010). Most studies have been conducted for urban environments, though, such as the extraction of roofs from airborne LiDAR data (Jochem et al., 2012).

In order to obtain optimal results in geological applications, numerous methodologies have been proposed in recent years which are specifically designed for the application on rock mass data (Riquelme et al., 2014). This diverging development is due to rock material commonly being of irregular shape, featuring varying amounts of surface roughness (on different spatial scales) and potentially occurring at any orientation within the data (Lato and Vöge, 2012).

The objective of our study is to develop a suitable workflow for the automated process of detecting planes in outcrop data to calculate their respective orientation, using 3D point clouds acquired by TLS. An algorithm for characterizing outcrops by detecting planes and determining their orientation (cf. Miernik et al., 2013) is deployed. Based on this method, the following main research questions are investigated in detail:

- To what degree can spatial resolution (here: point density) of the TLS survey be reduced without compromising the quality of results?

- Can smoothing of the data prior to the segmentation further enhance the results generated by the current 3D outcrop characterization algorithm?

\footnotetext{
${ }^{*}$ Corresponding author
} 
By addressing these research questions, our study gives insights in advantageous scan resolution set-ups and a robust method for plane segmentation, and aims at supporting similar projects of geological outcrop characterization.

\section{STATE OF THE ART}

Early studies on the identification of discontinuity surfaces apply a least squares method to subsets of points in order to determine the best-fit plane through planar regression (Abellán et al., 2006; Fernández, 2005). In the past years, the calculation of normal vectors in association with point cloud subsets has become a widely accepted methodology (Riquelme et al., 2014). Most methods currently conduct the analysis on simplified 2.5D surface models, for example by using triangulated irregular networks (TINs) (Riquelme et al., 2014). Specifically in geological applications, however, this implication of converting 3D point clouds into 2.5D data might lead to an unwanted loss in detail and accuracy, as the representation of overhangs and concavities is not supported (Mah et al., 2011). Mah et al. (2011), in this context, present a workflow involving the transformation of the point cloud into a 3D triangular mesh.

Riquelme et al. (2014) recently proposed a method using the 3D information of the point cloud itself by examining every point and its local neighbors, respectively. Throughout the workflow, individual points are tested for coplanarity in a specified neighborhood based on their local curvature, which is determined by means of a Principal Component Analysis (PCA). Subsequently, the calculated PCA is used to adjust a best-fit plane to the found subsets of coplanar points. A principal orientation is then assigned to each point by kernel density estimation and, eventually, the points are clustered in space. The aim of the authors is to derive geometrical parameters, such as planar orientation, from true 3D information, i.e., based directly on the point cloud. Similarly, using true 3D information, and thus avoiding prior triangulation, is the motivation for Miernik et al. (2013) to apply a methodology that directly extracts geometric outcrop information from the 3D point cloud. It is based on robust local plane fitting and subsequent region growing. The algorithm developed in the frame of Miernik et al. (2013) is improved and applied in this study and will be explained in detail in Section 3.

Lato and Vöge (2012) include a data processing step in their approach prior to the actual segmentation with the purpose of eliminating surface roughness artefacts, which largely depend on the scale of the study object, its geometric features, and also the data capturing method. Lato and Vöge (2012) additionally mask out sharp edges to exclude large deviations in normal direction from the actual segmentation process. In their workflow, data smoothing is a powerful means of generating a more thorough representation of planar surfaces in the outcrop data and is subsequently also examined in our study.

Regarding spatial resolution and scale for geological applications, the surveying of rather large areas is likely to be desired. Point clouds of lower resolution (here: point density) would allow for a significant reduction in acquisition time and subsequent effort of computing and processing. Buckley et al. (2008) point to the option of resampling data to a level adequate for the scale of an outcrop study.

As outlined in this section, aspects of the given research questions have been proposed in various publications and implemented in the respective workflows. However, a comprehensive investigation on the effects of reduced density and smoothing of 3D point cloud data has not yet been undertaken. Against this background, our research aims to address these aspects in an integrated study.

\section{METHODS}

The workflow designed for addressing the previously given research questions is illustrated in Fig. 1. The individual steps are described in detail subsequent to the description of the study site and the procedure of data acquisition and processing.

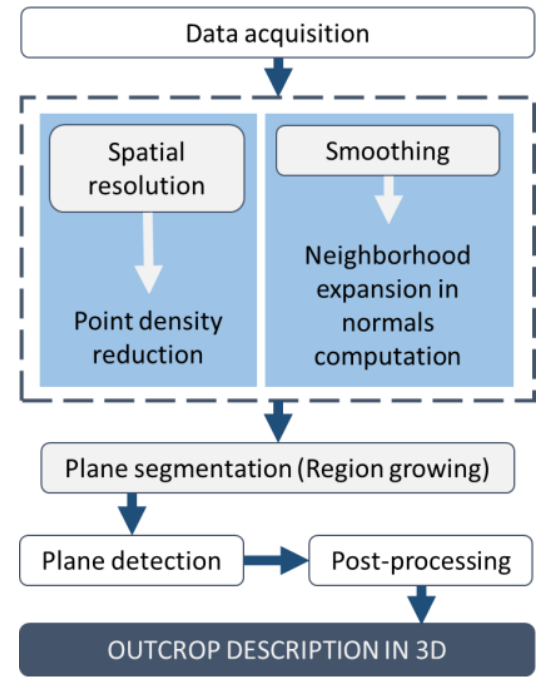

Figure 1. Workflow of studying automatic plane detection in geological outcrop data using 3D point clouds directly

\subsection{Study site and dataset}

Point cloud data for the study are acquired on an outcrop close to Kalávrita on the Northern Peloponnesian peninsula in Greece (Fig. 2) using the TLS system Riegl VZ-6000 with full-waveform online echo detection. The outcrop is scanned from three positions at a laser pulse repetition rate of $300 \mathrm{kHz}$ and a point

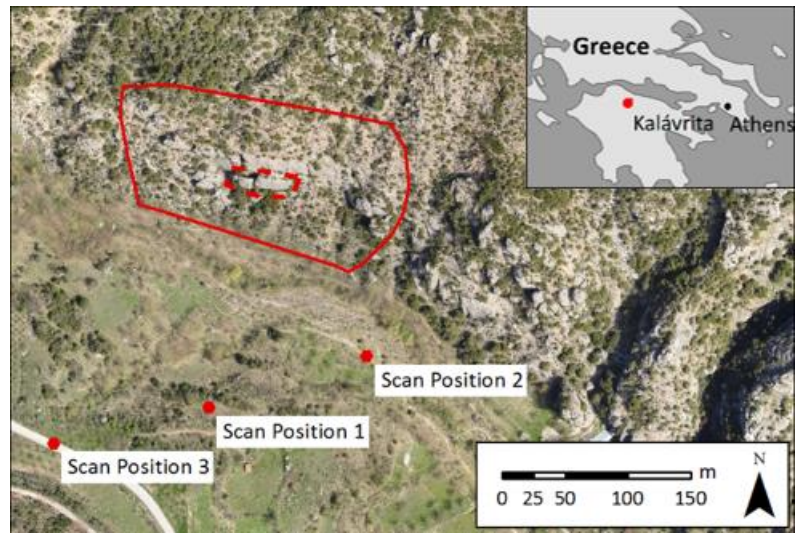

Figure 2. Location of the study site in Greece $\left(38.06^{\circ} \mathrm{N}\right.$, $22.13^{\circ} \mathrm{E}$ ) with the area of interest (red frame) and the extract used for the detailed study (red dashed frame). Orthophoto derived from aerial imagery acquired by an eBee (2015)

spacing of $3 \mathrm{~mm}$ at $10 \mathrm{~m}$ distance. This high resolution is obtained in order to address the corresponding question of research regarding spatial resolution. Acquiring data from varying positions both horizontally and vertically aims at minimizing occlusion and therefore missing data. However, parts of the dataset still 
contain occlusions due to limited accessibility to areas surrounding the outcrop. Nonetheless, the study area is captured well and the three scans are considered suitable for our research.

The captured LiDAR data is georeferenced directly via scannerintegrated inclination and orientation sensors and a high-end external RTK GNSS. This provides a 3D accuracy of $0.03 \mathrm{~m}$ for the scan positions.

Scanning and data processing are conducted using RiSCAN PRO (RIEGL, 2015). Processing steps comprise the registration as well as revision and manual outlier cleaning of the dataset. In order to improve co-registration of the already georeferenced point clouds of the three scan positions, RiSCAN PRO multi-station adjustment is conducted with a final ICP standard deviation of errors of $0.013 \mathrm{~m}$. Further processing of the data as preparation for the application of the plane detection algorithm is undertaken in the modular program system 'Orientation and Processing of Airborne Laser Scanning data' (OPALS, Pfeifer et al., 2014), especially regarding the computation of surface normals as a basis for the plane segmentation by estimating point based local plane information considering the full 3D neighborhood of coordinates.

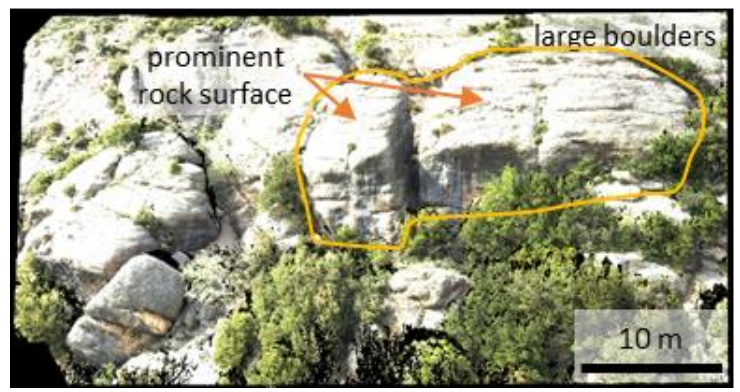

Figure 3. RGB-colored point cloud of the subsample for testing the algorithm before application to the whole study area

The area of interest for the outcrop study is delineated in red in the overview map of the study area in Fig. 2. The dashed line marks the extract used for the individual steps of investigating the research questions. The extract contains large boulders with prominent rock surface planes (Fig. 3). Given their geometrical features, including bedding planes and weathering surfaces as well as fractures, the extract is a well suited study sample for the intended purpose of plane detection in rock surfaces. Ultimately, the developed workflow is applied to the dataset for the entire outcrop. Furthermore, the determination of plane sets, i.e. sets of similar orientation, is applied to the area of interest as a whole.

\subsection{Plane detection}

The detection of planes in the point cloud is conducted by applying an algorithm developed in the frame of a study by Miernik et al. (2013). The algorithm is implemented in a standalone C++ tool and performs region growing segmentation by selecting those points with the lowest curvature values in respect to their neighborhood as seeds. Subsequent segment growing is done in a specified neighborhood and limited by a maximum surface normal angle deviation.

Prior to the segmentation, local normal information is estimated by applying a robust plane fit to the nearest neighbors in a specified neighborhood radius. Herein, for enhanced approximation of the local environment of a point, a strategy of additional inversed distance weighting is used. The output includes the standard deviation returned for the plane fit, which is used in the algorithm and subsequently addressed as plane sigma.
The segmentation algorithm begins by computing the curvature for each point based on the respective eigenvalue ratio $C_{e}$ according to Weinmann et al. (2014):

$$
C_{e}=\frac{e_{1}}{e_{1}+e_{2}+e_{3}}
$$

where $e_{1}<e_{2}<e_{3}$

Seed points are then listed ordered by increasing curvature, so that the actual segmentation will start with the point of lowest curvature and proceed along the list of subsequent seed points for points that have not been segmented already to previous objects. The algorithm then checks a specified number of points in the neighborhood of the seed point for defined homogeneity criteria. These criteria are listed below according to the workflow in the segmentation algorithm.

1) Angular deviation: deviation of a point's normal vector to the respective seed point's normal. In the further course of region growing, the angle criterion is assessed based on the deviation to the plane adjusted to points already assigned to the current segment.

2) Distance to the plane and to segmented points: the distance of the point to the plane in perpendicular direction as well as the direct distance to the segmented points is checked against specified maximum distances.

3) Curvature and plane sigma as indicator of roughness consideration of the curvature and plane sigma values of a point, which prohibits the exceedance of specified thresholds. Both parameters are determined as percentile thresholds, whereas the values of plane sigma can be understood as the roughness of the local plane surface.

A segment is completed when no more unclassified points are found in the neighborhood or the specified maximum size of segments is reached. Hereafter, the orientation of the plane adjusted to the segment is determined. This principal orientation, i.e. azimuth and dip values, is assigned to every single point classified as part of the respective plane segment. Points that are not segmented as part of a plane or compose a plane in a number that does not exceed a specified threshold are discarded.

\subsection{Validation}

As reference data for validation, strike and dip values of several plane sets, i.e. numerous planar surfaces, may be determined manually at the survey site. In reference to Mah et al. (2011), though, the assumption is adopted that planar orientations derived from TLS acquired point cloud data may be of higher accuracy than orientation values determined by manual field measurements. Against this background and in respect of the purpose of this study, a reasonable means of validating the results of the study is the use of reference planes manually designated in the point cloud. As reference, rock surface planes are chosen based on expert knowledge (Fig. 4a). In the further course of the study, the focus will be on planes 01 and 02 , as these comprise different geometric characteristics and, thereby, pose a suitable sample for all five reference planes. Given uniform slope and surface characteristics, similar validation results are generated for the reference planes 02,04 , and 05 . This also accounts for planes 01 and 03 , both exhibiting a more vertical, less fractured character.

For each of the reference planes, the respective segment IDs of corresponding points are extracted from the segmented point cloud. The plane segmentation is then assessed based on the number of segments within a reference plane and the number of points 
in each segment, which represents its respective size. The assumption is that a small number of segmented planes of relatively large size in respect to the total reference plane show a higher validity. Following, validity will be addressed in terms of congruency, which is determined by the percentage of points in the largest segment relative to the number of points in the corresponding reference plane. However, it is to be emphasized that the reference planes as overall surface planes partly contain distinctive fractures as becomes apparent only on close up inspection of, for example, reference plane 02 as illustrated in Fig. 4b. Thus, the congruency with the respective reference plane might be significantly reduced.

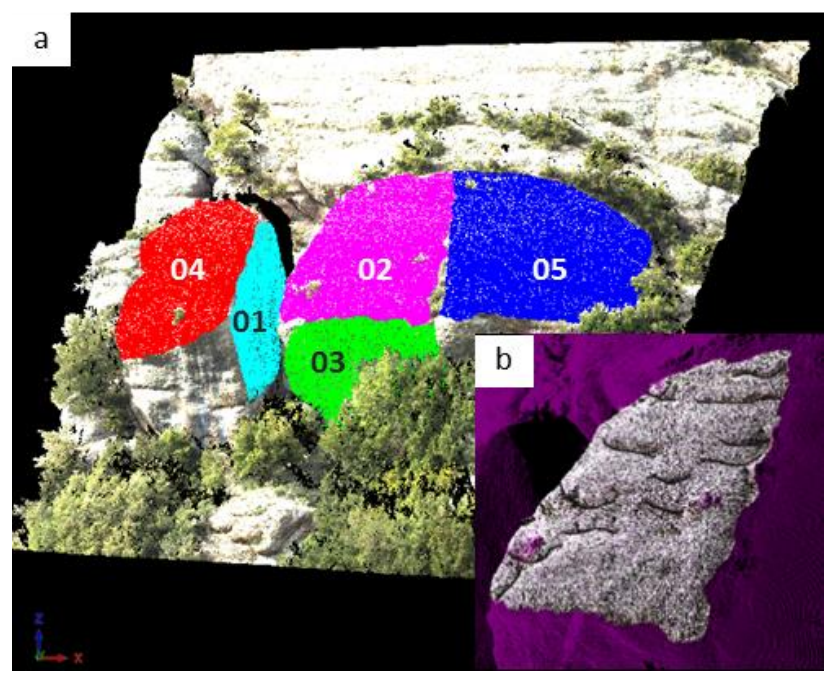

Figure 4. Designated reference planes for the studied data extract (a). Close up of reference plane 02 with designated fractures in the weathered rock surface (b)

\subsection{Spatial resolution}

In order to determine the spatial resolution required for adequate segmentation results, datasets acquired at lower spatial resolutions are simulated from the high-resolution raw data. For that purpose, an algorithm is applied that produces a point cloud by using only every $\mathrm{n}^{\text {th }}$ point of the original dataset, i.e. every $\mathrm{n}^{\text {th }}$ point in the point cloud. By this, the point cloud density is reduced progressively. The plane segmentation is conducted for various percentages of the original point density $(50 \%, 20 \%$, $10 \%$, i.e. step widths $n=2,5,10$ ).

\subsection{Smoothing}

Rock surfaces feature varying amounts and scales of surface roughness that are also captured by laser scanning surveys. Furthermore, small-scale roughness artefacts may derive from remaining errors owing to the registration of various scans from different positions (Lato and Vöge, 2012). It might, hence, be expedient to smooth the surfaces of input data before applying a plane detection algorithm. To achieve such smoothing, the data processing step of computing normals is modified by increasing the search radius and the number of neighboring points considered. Fig. 5 displays the effect of an increased search radius on local normals. The normals are oriented in a much more uniform direction, which directly influences the angle criterion in the segmentation algorithm. This emphasizes the implication for the performance of the plane detection.
As processing time increases exponentially with the expansion of the search radius and given the previously assessed efficiency of lowered spatial resolution, the data for the normals estimation and subsequent segmentation are used at a point density of $20 \%$.
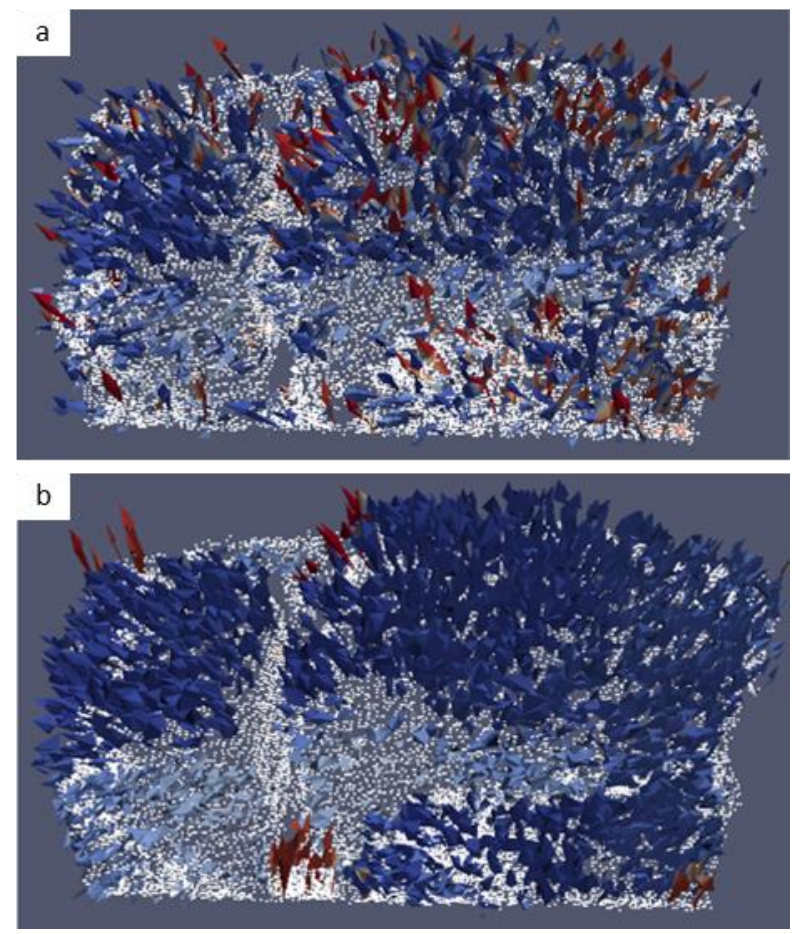

Figure 5. Visualization of normals in ParaView (Ayachit, 2015) computed with a search radius of $0.2 \mathrm{~m}$ (a) and $5 \mathrm{~m}$ (b)

\subsection{Post-processing of segmented data}

Benefits for a geological application arising from plane detection in the unorganized point cloud need to be assessed in specific post-processing steps in order to interpret and visualize the character of an outcrop. A processing step we included addresses the mentioned topic of building sets of discontinuities. It is implemented by grouping segments of similar orientation in clusters and thereby generating affiliations to plane sets as a further output feature. In the process, random RGB colors are assigned to each built set, so that the outcrop ultimately can be visualized not only according to segments but also as plane sets. The aggregation of plane segments into families of plane orientation is undertaken sequentially by a comparison of a segment's orientation to the mean orientation of segments in already built families. If a segment cannot be assigned to an existent family, it is considered an additional orientation set and a new family is created.

\section{RESULTS AND DISCUSSION}

\subsection{Plane detection}

An overall assessment of the outcrop characteristics and thorough consideration of the parametrization of the segmentation step lead to the conclusion that the optimal parameters for the application of the 3D outcrop characterization algorithm on the survey data are to be set as summarized below (Tab. 1). Thereby obtained plane segments are visualized in Fig. 6 . 


\begin{tabular}{|l|c|}
\hline Parameter description & Value \\
\hline Maximum angle between the normal vectors $\left[{ }^{\circ}\right.$ ] & 20.0 \\
Maximum distance of point to the plane [m] & 0.3 \\
Maximum distance of point to the segmented & 0.3 \\
points [m] & 75.0 \\
Maximum allowed curvature as percentile [\%] & 90.0 \\
Point selection threshold as percentile of rough- & \\
ness [\%] & 2,000 \\
Minimum segment size for output [no. of points] & \\
\hline
\end{tabular}

Table 1. Optimized parametrization for the data basis of the study

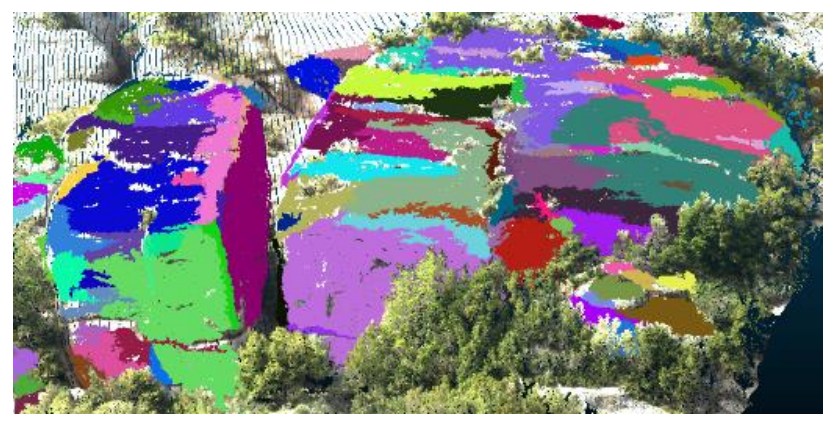

Figure 6. Result of plane segmentation according to optimized parametrization for the data basis of the study (Tab. 1) fit onto true color display

\subsection{Validation}

By validating the plane segments computed using the plane segmentation algorithm against reference planes 01 and 02 , congruencies of $93.0 \%$ and $30.9 \%$ were obtained (Fig. 7). These results express the previously mentioned challenge of dealing with fractures in the designated reference planes, from which the high number of segments in reference plane 02 originates. In consequence of the rather even distribution of reference points on many segments, the dominant plane segment does not cover a major part of the reference plane and, thus, yields a low congruency.
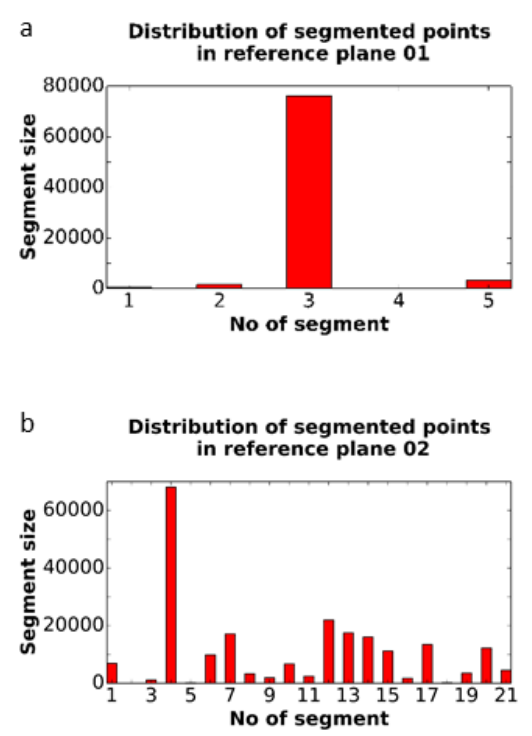

Figure 7. Bar plot segment number and number points per segmented plane. Shown are reference plane 01 (a) and 02

(b)
This result points at a further differentiation of surfaces being reasonable, for example regarding bedding planes or fractures. Accordingly, the designation of planes aimed at depends on the objective of a study and the corresponding geological question of research.

The discrepancy of the validation results in the presented study was intentionally not overcome by means of aggregating neighboring planes as would be done, for example, for building modeling by roof detection (Dorninger and Northegger, 2007). In fact, for the geological description of outcrops it is more significant to classify individual plane patches in discontinuity sets, i.e. families of plane patches with similar orientation (Garcia-Cortes et al., 2012). Therefore, rather low percentages of congruency and a high number of plane segments per reference plane were accepted for further processing. The mere detection of planes, against this background, is considered well performed. An implementation of building plane sets is introduced in the section on post-processing of segmented data.

\subsection{Spatial resolution}

The validity assessment (Tab. 2) shows that the performance of the algorithm is not significantly compromised by a reduction of spatial resolution, but rather fluctuating around the value achieved with the original point cloud density. The results obtained by a segmentation of a point cloud of only $10 \%$ of the highresolution data are visualized in Fig. 8.

\begin{tabular}{|c|c|c|c|c|c|}
\hline Percentage of & \multicolumn{5}{|c|}{ Reference plane } \\
data volume & 01 & 02 & 03 & 04 & 05 \\
\hline $100 \%$ & 93.0 & 30.9 & 78.3 & 31.6 & 33.0 \\
& $(5)$ & $(21)$ & $(7)$ & $(21)$ & $(28)$ \\
\hline $50 \%$ & 94.6 & 34.4 & 80.3 & 53.7 & 34.6 \\
& $(5)$ & $(16)$ & $(5)$ & $(10)$ & $(28)$ \\
\hline $20 \%$ & 92.4 & 27.9 & 76.5 & 56.8 & 33.6 \\
& $(7)$ & $(18)$ & $(5)$ & $(21)$ & $(23)$ \\
\hline $10 \%$ & 91.5 & 30.2 & 81.4 & 58.2 & 40.6 \\
& $(5)$ & $(16)$ & $(6)$ & $(17)$ & $(25)$ \\
\hline
\end{tabular}

Table 2. Validity of planes for varying spatial resolutions in terms of congruency with the reference planes in \%, number of segmented planes in brackets

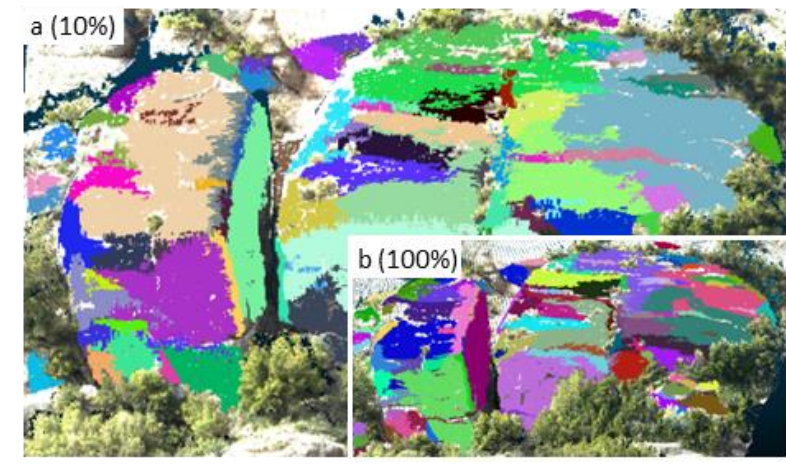

Figure 8. Result of plane segmentation for a point cloud with only $10 \%$ of the original density (a) compared to results based on the original point cloud (b)

Reduced point densities hardly affect the segmentation results, which allows for the conclusion that the algorithm is robust against changing point spacing and still shows high accuracy even when applied to data acquired at a much lower spatial resolution. 
Moreover, facing extremely long processing times and an enormous demand on computer hardware for the processing of large point clouds, this puts the processing of rather large areas of interest into perspective. In this respect, even for detailed datasets at hand, it may be advisable to greatly increase their point spacing for the mere process of plane detection. This greatly promotes the suggestion of Buckley et al. (2008) that outcrop studies predominantly do not require very high point densities. The reduction of input data to only $20 \%$ of the original point cloud enabled reliably computing the full workflow for the total area of interest on conventional computer hardware. Obtained results are displayed in Fig. 9a.

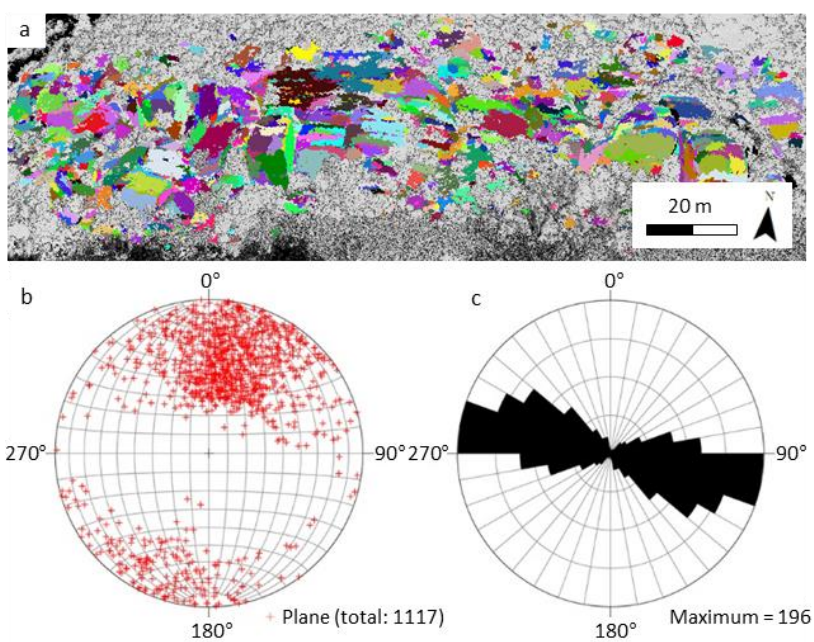

Figure 9. (a) Visualization of plane segments in area of interest fit into point cloud of scan position 01 (b) Equal area stereographic projection of poles to planes (c) Rose plot of strike directions

Fig. 9b illustrates the orientation character of the outcrop in a stereographic projection of the results. Herein, the generated plane segments are visualized according to their respective poles, created by projecting their surface normals into the lower hemisphere. The result clearly shows a clustering of poles to planes in the upper part. This indicates a main orientation of the rock surfaces facing in SSW-direction. Respective inclinations range from $20^{\circ}$ to $90^{\circ}$, which indicates possible occlusion effects of subhorizontal planes. Further planes, as positioned in the lower part of the stereo net, are oriented in opposite directions. These segments represent overhangs and, partly, the backside of outstanding rocks. The multitude of poles being located in the outer parts of the stereographic projection refers to prominent vertical fracture sets.

The corresponding rose plot (Fig. 9c) was generated from the azimuth information per segment and illustrates the strike character of the outcrop. The largest cluster is built between $90^{\circ}$ and $100^{\circ}$ and comprises 196 planes. Thus, the strike of the rock surface runs mainly in WNW- and ESE-direction.

Fig. 10 demonstrates the outcome of assigning segmented planes to plane sets. This grouping of planes can pose as an effective way of visualizing plane sets, such as discontinuity families. However, a strong aggregation of plane information is involved in the currently applied procedure. Therefore, further development of the process of grouping planes into sets might consider extensions such as implementing the grouping of similarly oriented segments by the use of dynamic clustering.

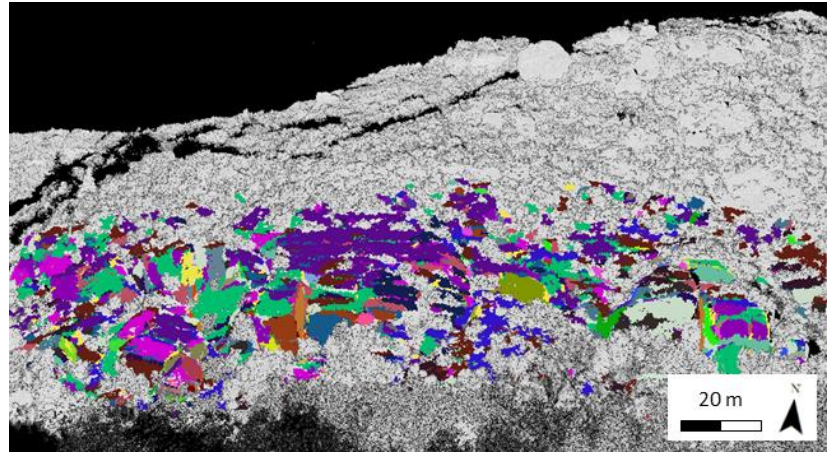

Figure 10. Visualization of plane sets in the area of interest fit into point cloud of scan position 01

\subsection{Smoothing}

Smoothing the input data prior to the actual segmentation by applying a larger neighborhood radius for normal computation resulted in a more general representation of (major) planes in the rock surfaces. This interpretation can be drawn from the results visualized in Fig. 11a. Segmented planes widely transcend unevenness in the rock surface and build significantly larger segments.

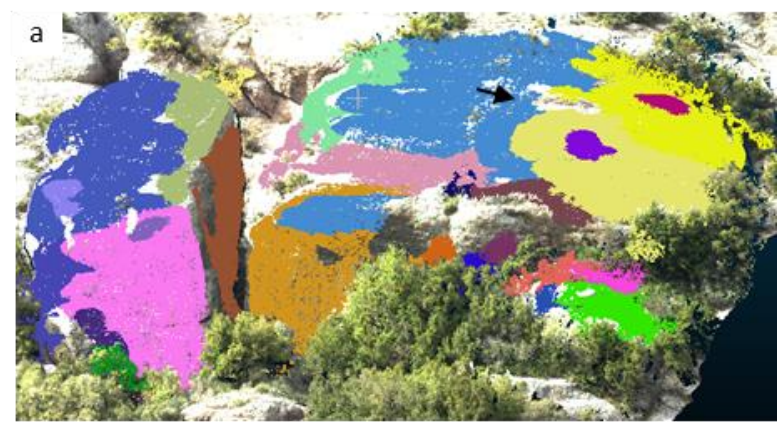

b

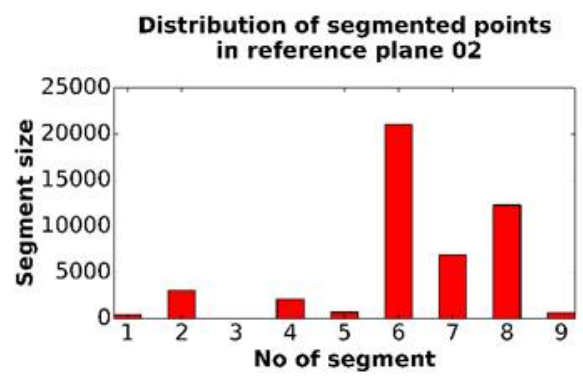

Figure 11. Result of plane segmentation for smoothed data. The black arrow marks the transcendence of the segment from reference plane 02 to 05 (a). Bar plot with number of points and distribution on segmented planes for reference plane 02 (b)

The bar plot addressing the validity in accordance with reference plane 02 (Fig. 11b) shows that a smaller total number of segments and a considerably higher congruency is yielded than for the results obtained based on normals computed in a smaller neighborhood (see also Fig. 7b). It should be noted, though, that this involves segments also transcending thresholds between aimed at planes. The black arrow in Fig. 11a marks this case between reference planes 02 and 05 . Hence, with the maximum applied search radius of $5 \mathrm{~m}$, the planar surfaces as referenced could not be fully achieved, although a significant increase in reference matching was observed for previously low congruencies of certain reference planes. Altogether, the results obtained support the 
assumption that smoothing of the data should be applied scaledependently.

Therefore, the adequate degree of smoothing should not be determined fully automatically. The search radius of local normals estimation rather needs to be adapted to the individual aims of analyses, acquisition settings, and outcrop characteristics. This requires skilled consideration from the user and it can have significant impact on the informative value achieved when results are to be used as basis for further analyses. For the geological setting and character of the studied outcrop, a search radius of $0.2 \mathrm{~m}$ was found to be effectual in order to ensure the generation of plane segments at an adequate level of detail. The neighborhood should be substantially decreased or increased for varying scales of outcrop analyses. This corresponds to the smoothing approach as implemented by Lato and Vöge (2012), which is to be set to a certain level interactively by the user.

\section{CONCLUSIONS}

This paper demonstrates that the applied methodology of 3D outcrop characterization represents a powerful approach to the automatic detection of planar surfaces in 3D outcrop data. In future work, validation by means of geological mapping of planes could ensure to determine tangible benefits more comprehensively. Against the background of the presented study, the results obtained by our workflow can be considered as accurate, seeing that the primary objective of detecting rock surface planes and deriving respective orientations from unorganized $3 \mathrm{D}$ point cloud data is met.

Our results show that the developed workflow is robust regarding varying target objects, different scales of study sites, and varying data capturing setups. As initially stated in this context, it is of particular significance that the presented algorithm supports the irregular shape of objects, varying surface roughness and planes potentially being oriented at any direction in the data. For a prospective useful deployment of the algorithm, the statement of Buckley et al. (2008) comes to bear, emphasizing the importance of profound understanding of geology for the effective solution of a research question.

Depending on the characteristics of a study area, data gaps potentially occur due to occluded LiDAR measurements. Missing data might be filled, for example, by using unmanned aerial systems (UAS) for image acquisition and subsequently generating complementary point cloud data through photogrammetric approaches. Similarly, UAS-borne laser scanning can be considered a complementary approach increasingly applied in geoscientific research (Wallace et al., 2016; Lumme et al., 2008), also offering new opportunities to capture complex geological sites comprehensively.

Finally, to further develop the existent methodology, additional aspects such as the distinction of rock material in outcrops with heterogeneous composition might be included. For this purpose, it is to be investigated in what way, for example, LiDAR backscatter characteristics or waveform recordings can be implemented in the plane segmentation algorithm in order to differentiate planes into individual rock specific plane segments. Separability of rocks and the combination of intensity values with geometrical information has recently been presented by Penasa et al. (2014) and shows potential for further research and implementation in the presented 3D outcrop characterization tool.

\section{ACKNOWLEDGEMENTS}

We would like to thank the Department of Petroleum Geosciences Engineering of the University of Stavanger for valuable input on on-site geology and, together with Total E\&P Norge AS, for their support in realizing the field work in Greece. We thank senseFly for the provision of an eBee for on-site aerial image acquisition and Michael Ebner for the survey and generation of the orthophoto. Many thanks also to Luisa Griesbaum and Michael Ebner for helping in the field campaign as well as with data processing. Furthermore, we thank Jörn Profe and Sebastian Bechtold for their contributions in implementing the algorithm.

\section{REFERENCES}

Abellán, A., Oppikofer, T., Jaboyedoff, M., Rosser, N.J., Lim, M., Lato, M.J., 2014. Terrestrial laser scanning of rock slope instabilities. Earth Surface Processes and Landforms, 39, pp. 8097.

Abellán, A., Vilaplana, J.M., Martínez, J., 2006. Application of a long-range Terrestrial Laser Scanner to a detailed rockfall study at Vall de Núria (Eastern Pyrenees, Spain). Engineering Geology, 88, pp. 136-148.

Ayachit, U., 2015. The ParaView Guide. Kitware Inc.

Barnea, S., Filin, S., 2013. Segmentation of terrestrial laser scanning data using geometry and image information. ISPRS Journal of Photogrammetry and Remote Sensing, 76, pp. 33-48.

Beraldin, J.-A., Blais, F., Lohr, U., 2010. Laser Scanning Technology. In: Vosselmann, G., Maas, H.-G. (Eds.), Airborne and Terrestrial Laser Scanning. Whittless Publishing, Dunbeath, pp. $1-42$.

Buckley, S.J., Enge, H.D., Carlsson, C., Howell, J.A., 2010. Terrestrial laser scanning for use in virtual outcrop geology. Photogrammetric Record, 25, pp. 225-239.

Buckley, S.J., Howell, J.A., Enge, H.D., Kurz, T.H., 2008. Terrestrial laser scanning in geology: data acquisition, processing and accuracy considerations. Journal of the Geological Society, 165 , pp. $625-638$.

Dorninger, P., Northegger, C., 2007. 3D Segmentation of Unstructured Point Clouds for Building Modelling. International Archives of Photogrammetry, Remote Sensing and Spatial Information Sciences, 36, pp. 191-196.

Fekete, S., Diederichs, M., 2013. Integration of threedimensional laser scanning with discontinuum modelling for stability analysis of tunnels in blocky rockmasses. International Journal of Rock Mechanics and Mining Sciences, 57, pp. 11-23.

Fernández, O., 2005. Obtaining a best fitting plane through 3D georeferenced data. Journal of Structural Geology, 27, pp. 855858 .

Fisher, J.E., Shakoor, A., Watts, C.F., 2014. Comparing discontinuity orientation data collected by terrestrial LiDAR and transit compass methods. Engineering Geology, 181, pp. 78-92.

Fonstad, M.A., Dietrich, J.T., Courville, B.C., Jensen, J.L., Carbonneau, P.E., 2013. Topographic structure from motion: a new development in photogrammetric measurement. Earth Surface Processes and Landforms, 38, pp. 421-430. 
Garcia-Cortes, S., Galan, C.O., Arguelles-Fraga, R., Diaz, A.M., 2012. Automatic detection of discontinuities from 3D point clouds for the stability analysis of jointed rock masses. 18th International Conference on Virtual Systems and Multimedia. IEEE, Milan, pp. 595-598

Höfle, B., Rutzinger, M., 2011. Topographic airborne LiDAR in geomorphology: A technological perspective. Zeitschrift für Geomorphologie, 55, pp. 1-29.

Jochem, A., Höfle, B., Wichmann, V., Rutzinger, M., Zipf, A., 2012. Area-wide roof plane segmentation in airborne LiDAR point clouds. Computers Environment and Urban Systems, 36, pp. 54-64.

Lato, M.J., Vöge, M., 2012. Automated mapping of rock discontinuities in 3D lidar and photogrammetry models. International Journal of Rock Mechanics and Mining Sciences, 54, pp. 150-158.

Lumme, J., Karjalainen, M., Kaartinen, H., Kukko, A., Hyyppä, J., Hyyppä, H., Jaakola, A., Kleemola, J., 2008. Terrestrial laser scanning of agricultural crops. In: The International Archives of the Photogrammetry, Remote Sensing and Spatial Information Sciences, Beijing, China, Vol. XXXVII. Part B5, pp. 563-566.

Mah, J., Samson, C., McKinnon, S.D., 2011. 3D laser imaging for joint orientation analysis. International Journal of Rock Mechanics and Mining Sciences, 48, pp. 932-941.

Miernik, G., Profe, J., Höfle, B., Kissner, T., Emmerich, A., Bechstädt, T., Zühlke, R., 2013. Modelling fractured reservoirs from LiDAR derived digital outcrop models (DOMs). Proceedings of the 30th IAS Meeting of Sedimentology, pp. 2.

Penasa, L., Franceschi, M., Preto, N., Teza, G., Polito, V., 2014. Integration of intensity textures and local geometry descriptors from Terrestrial Laser Scanning to map chert in outcrops. ISPRS Journal of Photogrammetry and Remote Sensing, 93, pp. 88-97.

Pfeifer, N., Mandlburger, G., Otepka, J., Karel, W., 2014. OPALS - A framework for Airborne Laser Scanning data analysis. Computers, Environment and Urban Systems, 45, pp. 125-136.

RIEGL, 2015. Operating \& Processing Software: RiSCAN PRO for RIEGL 3D Laser Scanners, pp. 3.

Riquelme, A.J., Abellán, A., Tomás, R., Jaboyedoff, M., 2014. A new approach for semi-automatic rock mass joints recognition from 3D point clouds. Computers \& Geosciences, 68, pp. 38-52.

Sturzenegger, M., Stead, D., 2009. Quantifying discontinuity orientation and persistence on high mountain rock slopes and large landslides using terrestrial remote sensing techniques. Natural Hazards and Earth System Sciences, 9, pp. 267-287.

Vosselman, G., Klein, R., 2010. Visualisation and Structuring of Point Clouds. In: Vosselmann, G., Maas, H.-G. (Eds.), Airborne and Terrestrial Laser Scanning. Whittless Publishing, Dunbeath, pp. $45-81$.

Wallace, L., Lucieer, A., Malenovský, Z., Turner, D., Vopenka, P., 2016. Assessment of Forest Structure Using Two UAV Techniques: A Comparison of Airborne Laser Scanning and Structure from Motion (SfM) Point Clouds. Forests, 7(3), 62.
Weinmann, M., Jutzi, B., Mallet, C., 2014. Semantic 3D scene interpretation: A framework combining optimal neighborhood size selection with relevant features. ISPRS Annals of the Photogrammetry, Remote Sensing and Spatial Information Sciences, Zurich, Switzerland, Vol. II-3, pp. 181-188. 